In the FTIR spectra, the peak at $1310 \mathrm{~cm}^{-1}$ is characteristic of the $\mathrm{C}-\mathrm{N}$ stretching mode in the $\mathrm{QBB}$ moiety, ${ }^{[11]}$ where $\mathrm{Q}$ and $\mathrm{B}$ denote the quinoid and benzenoid rings, respectively. The $1310 \mathrm{~cm}^{-1}$ peak, apart from showing a red shift to $1300 \mathrm{~cm}^{-1}$, gains substantial intensity. Along with this, a new feature appears at $1240 \mathrm{~cm}^{-1}$, which is assigned to $\mathrm{C}-\mathrm{N}^{+}$ stretching in the $\mathrm{BBB}$ moiety. We notice a small peak at $1374 \mathrm{~cm}^{-1}$ in emeraldine base, which diminishes gradually with an increase in the doping. The origin of this change is not clear to us at present; however, it provides a convenient way to monitor the extent of Lewis acid doping. The $1165 \mathrm{~cm}^{-1}$ peak has been identified as the vibrational mode for the $\mathrm{N}=\mathrm{Q}=\mathrm{N}$ group. In the spectra of weakly protonated samples, one observes two separate peaks at $1160 \mathrm{~cm}^{-1}$ and $1140 \mathrm{~cm}^{-1}$ and as the degree of protonation increases, those merge into one broad and intense feature, centered at $1140 \mathrm{~cm}^{-1}$. In our sample, the broad feature appearing at $1130 \mathrm{~cm}^{-1}$ has been assigned to the groups, such as $\mathrm{B}-\mathrm{N}^{+} Z-\mathrm{Q}$ or $\mathrm{B}-\mathrm{N}^{+} Z-\mathrm{B}$, where $Z$ denotes the $\mathrm{BF}_{3}$ moiety. There are two sharp, intense peaks at 1124 and $1084 \mathrm{~cm}^{-1}$, which are almost masked by the broad feature in this region, $850-1200 \mathrm{~cm}^{-1}$. Comparing it with the IR spectra of boron trifluoride-tetrahydrofuran $\left(\mathrm{BF}_{3}-\mathrm{THF}\right)$ adduct, where a similar broad band with sharp spikes at 1123.5 and $1084 \mathrm{~cm}^{-1}$ is observed, we attribute these features to one of the B-F stretching modes. However, we could not identify the B-N stretching modes and suspect those to be masked by other peaks.

In summary, we have synthesized a highly conducting polyaniline, doped with a Lewis acid, boron trifluoride. NMR, IR, and optical absorption spectra confirm the ability of this electron-deficient molecule to dope polyaniline very effectively, yielding a remarkably high conductivity over a wide temperature range.

Received: May 18, 2001

[1] I. Kulszewicz-Bajer, A. Pron, J. Abramowicz, C. Jeandey, J.-L. Oddou, J. W. Sobczak, Chem. Mater. 1999, 11, 552.

[2] A. G. MacDiarmid, J. C. Chiang, A. F. Richter, A. J. Epstein, Synth. Met. 1987, 18, 285 .

[3] A. G. MacDiarmid, J. C. Chiang, M. Halpern, W. Huang, S. Mu, L. Nanayakkara, D. Somasiri, W. Wu, S. I. Yaniger, Mol. Cryst. Liq. Cryst. 1985, 121, 173.

[4] A. J. Epstein, J. M. Ginder, F. Zuo, H. S. Woo, D. B. Tanner, A. F. Richter, M. Angelopoulos, W. S. Huang, A. G. MacDiarmid, Synth. Met. 1987, 21,63 .

[5] J. Joo, S. M. Long, J. P. Pouget, E. J. Oh, A. G. MacDiarmid, A. J. Epstein, Phys. Rev. B 1998, 57, 9567.

[6] C. B. Duke, E. M. Conwell, A. Paton, Chem. Phys. Lett. 1986, 131, 82.

[7] M. G. Roe, J. M. Ginder, P. E. Wigen, A. J. Epstein, M. Angelopoulos, A. G. MacDiarmid, Phys. Rev. Lett. 1988, 60, 2789.

[8] J. M. Ginder, A. F. Richter, A. G. MacDiarmid, A. J. Epstein, Solid State Commun. 1987, 63, 97.

[9] S. Stafström, J. L. Brédas, A. J. Epstein, H. S. Woo, D. B. Tanner, W. S. Huang, A. G. MacDiarmid, Phys. Rev. Lett. 1987, 59, 1464.

[10] W. D. Phillips, H. C. Miller, E. L. Meutterties, J. Am. Chem. Soc. 1959, 81 , 4496

[11] J. S. Tang, X. B. Jing, B. C. Wang, F. S. Wang, Synth. Met. 1988, 24, 231

\section{Large Area Photonic Crystal Slabs for Visible Light with Waveguiding Defect Structures: Fabrication with Focused Ion Beam Assisted Laser Interference Lithography**}

By Laura Vogelaar, Wietze Nijdam,

Henk A. G. M. van Wolferen, René M. de Ridder,

Frans B. Segerink, Eliane Flück, Laurens Kuipers, and Niek F. van Hulst*

Photonic crystals are novel materials with unique optical properties. ${ }^{[1]}$ The crystals have a periodic modulation of the refractive index. As a result, the dispersion of light will be described by a band structure analogous to those of electron waves atomic crystals. Under the right conditions a photonic crystal can exhibit a photonic bandgap: light in a certain range of optical frequencies is forbidden in the crystal. ${ }^{[2]}$ The existence of a photonic bandgap enables an unprecedented control of spontaneous emission and propagation. By locally disturbing the periodicity, a defect-associated photon state is created, which can be used to guide light.

A photonic crystal slab is a thin film with a two-dimensionally periodic refractive index modulation in the plane. ${ }^{[3,4]}$ In a photonic crystal slab the light is confined to the crystal plane by a classical slab waveguide construction. For in-plane wave vectors a bandgap can be created. Thus, the slab has applications in light guiding without the need for a full bandgap in all three dimensions. Optimal performance of the photonic crystal slab is expected when the slab is mirror symmetric in the vertical direction, i.e., when the material on both sides of the slab has the same refractive index at least in the region of the near-field tail of the in-plane propagating light. ${ }^{[5]}$

Photonic crystals are fabricated by periodically arranging materials with highly dissimilar refractive indexes. To obtain a bandgap in the visible the periodicity of the index modulation

[*] Prof. N. F. van Hulst, L. Vogelaar, F. B. Segerink, E. Flück, Dr. L. Kuipers

Applied Optics Group

Department of Applied Physics and $\mathrm{MESA}^{+}$Research Institute University of Twente

PO Box 217, NL-7500 AE Enschede (The Netherlands)

E-mail: N.F.vanhulst@tn.utwente.nl

W. Nijdam

Aquamarijn Micro Filtration

PO Box 217, NL-7500 AE Enschede (The Netherlands)

H. A. G. M. van Wolferen, Dr. R. M. de Ridder

Lightwave Devices Group

Department of Electrical Engineering and $\mathrm{MESA}^{+}$Research Institute

University of Twente

PO Box 217, NL-7500 AE Enschede (The Netherlands)

[**] The authors gratefully acknowledge Christian Hermann, Heiko Pittner, and Prof. Dr. Ortwin Hess of Theoretical Quantum Electronics, DLR Stuttgart (Germany) for preliminary calculations, Bert Otter and Mark Smithers for SEM images, Lucie Hilderink for performing thermal oxidation, Stein Kuiper and Herbert Wormeester for useful discussions, and Wouter Rensen for graphics. This research is part of the strategic research orientation on Advanced Photonic Structures of the MESA ${ }^{+}$Research Institute. The work is financially supported by the "Stichting voor Fundamenteel Onderzoek der Materie (FOM)", which is financially supported by the "Nederlandse organisatie voor Wetenschappelijk Onderzoek (NWO)". 
has to be in the submicrometer range, $<350 \mathrm{~nm}$. For the fabrication of photonic crystal slabs electron beam lithography has been by far the most frequently used technique, since it is one of the few techniques that fulfills the accuracy requirements on these length scales. ${ }^{[6]}$ However, due to its "direct sequential write" character e-beam lithography is time consuming for large area structures. It would be highly advantageous to define the entire periodic pattern at once. Laser interference lithography (LIL) has proven its ability to generate uniform, two-dimensional, periodic submicrometer patterns over very large areas (in the order of $\left.\mathrm{cm}^{2}\right),{ }^{[7,8]}$ while holographic lithography can produce three-dimensional photonic crystals. ${ }^{[9]} \mathrm{A}$ combination of large area patterning in two dimensions with LIL, and local patterning with a direct writing method, e.g., a focused ion beam (FIB) ${ }^{[10]}$ is ideal for future fabrication of photonic crystal slabs with arbitrarily shaped light-guiding defects.

Here, we present a combination of LIL with FIB-assisted deposition. Highly uniform freestanding photonic crystal slabs have been fabricated, extending over areas as large as a few hundred micrometer squared, with the possibility to introduce any kind of defect structure. In this way we realized, what is to our knowledge, the largest photonic crystal slab $(100 \mu \mathrm{m} \times$ $4 \mathrm{~mm}$ ) for visible radiation. Long line defects, extending over more than $1 \mathrm{~mm}$, were also introduced.

In our photonic crystal slabs $\mathrm{Si}_{3} \mathrm{~N}_{4}(n=2.16$ at $\lambda=670 \mathrm{~nm})$ and air acted as high and low index material, respectively. A triangular lattice of air holes in the $\mathrm{Si}_{3} \mathrm{~N}_{4}$ layer was fabricated in order to obtain a bandgap for transverse-electric (TE) modes (polarization in the plane of the crystal).${ }^{[1]}$ By making the slab freestanding, i.e., embedded in air on both sides, we obtain the desired vertical mirror symmetry of the crystal slab.

A schematic representation of the procedure to fabricate the photonic crystal slabs with light-guiding defects is depicted in Figure 1. The first step in the procedure is the generation of a periodic pattern with LIL. A $300 \mathrm{~nm}$ thick photo resist layer is exposed to the interference pattern of two obliquely incident coherent beams of ultraviolet (UV) light. After exposure and development the photo resist will duplicate the fringe pattern formed by the two incident beams. The period of the pattern is controlled with the incident angles. To create patterns that are periodic in two dimensions, the photo resist layer is exposed twice. Between exposures the substrate is rotated around the surface normal. With intermediate rotations of $90^{\circ}$ and $60^{\circ}$, square and triangular lattices are generated, respectively. After exposure to a certain dose of UV-light the photo resist layer will develop into a pattern of dots. A typical result of a triangular dot pattern in photo resist made with LIL is shown in Figure 2a.

The desired interference pattern is that of the two direct beams. However, their reflections from the underlying substrate also expose the photo resist. The reflections cause an additional, unwanted fringe modulation perpendicular to the substrate plane. This additional exposure of the resist can destroy the dot pattern. The influence of unwanted reflections
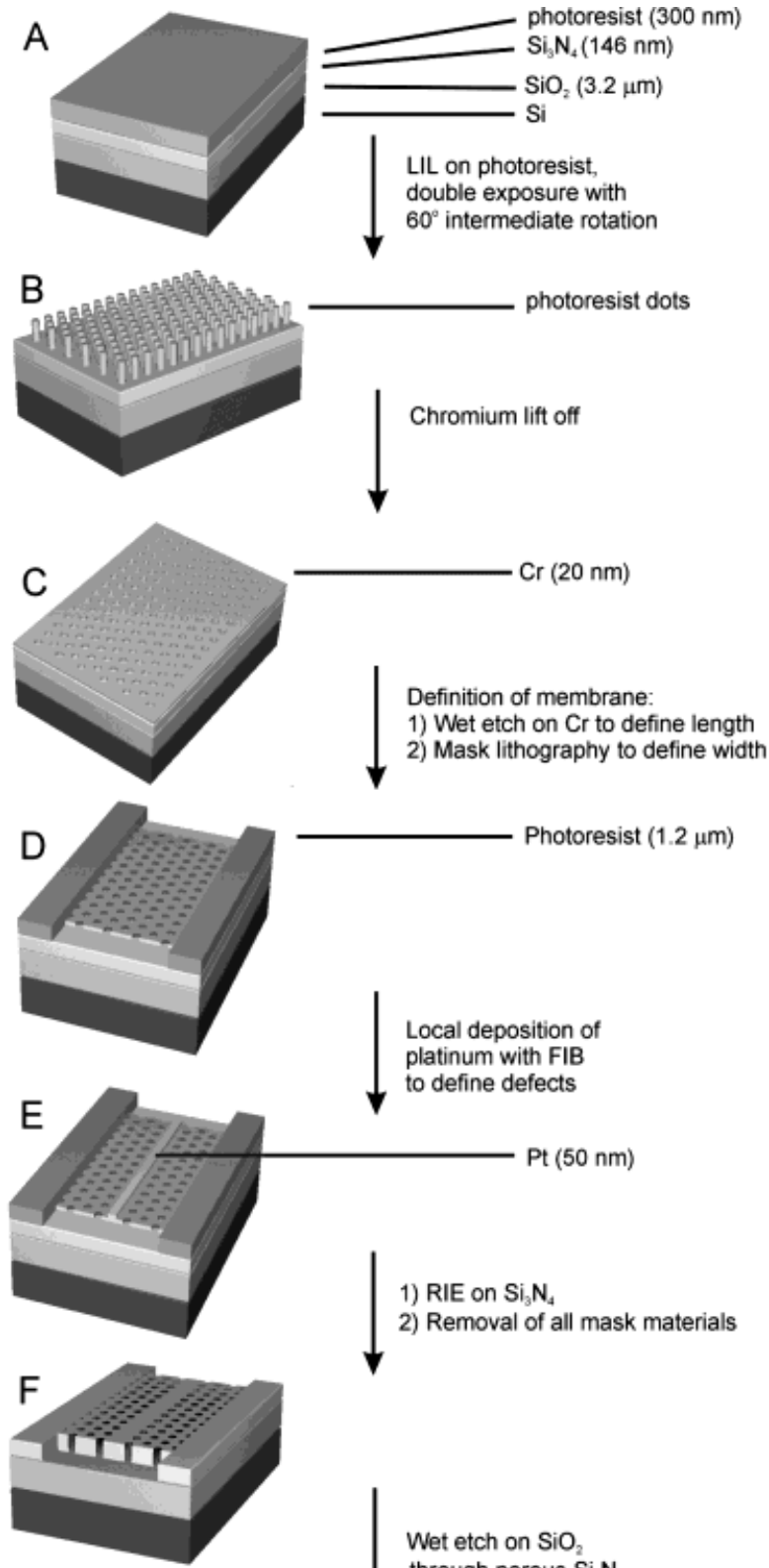
$\downarrow$

RIE on $\mathrm{Si}_{3} \mathrm{~N}$ Removal of all mask materials

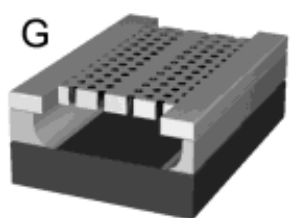

Wet etch on $\mathrm{SiO}$ through porous $\mathrm{Si}_{2} \mathrm{~N}$

Fig. 1. Schematic representation of the fabrication procedure, which combine large area patterning through LIL and local introduction of defect structures through FIB-assisted deposition. The dimensions in these pictures are chosen for illustrative clarity and are not to scale. Note that a slab of $100 \mu \mathrm{m} \times 4 \mathrm{~mm}$ contains over 4.5 million holes. A photo resist layer on top of a $\mathrm{Si}_{3} \mathrm{~N}_{4}(146 \mathrm{~nm})$, $\mathrm{SiO}_{2}(3.2 \mu \mathrm{m})$, and $\mathrm{Si}$ layer stack (A) is converted into a large area triangular dot pattern with LIL (B). By chromium lift off a thin, highly selective etch mask containing holes is formed (C). Next, the etch mask is divided into smaller sections by mask lithography (D). Local deposition of platinum is used to define defect structures of any shape in the photonic crystal slabs. Here, we demonstrate the introduction of a line defect (E). By RIE the structure is etched into the $\mathrm{Si}_{3} \mathrm{~N}_{4}$ layer, after which $\mathrm{Pt}, \mathrm{Cr}$, and photo resist are removed $(\mathrm{F})$. By a wet etch through the holes the underlying $\mathrm{SiO}_{2}$ layer is removed and the free-standing $\mathrm{Si}_{3} \mathrm{~N}_{4}$ photonic crystal slabs are formed $(\mathrm{G})$. 
for a given incident angle is minimized by adjusting the composition and thickness of the layers in the substrate. However, the thickness of the $\mathrm{Si}_{3} \mathrm{~N}_{4}$ layer that will form the photonic crystal slab, can not be freely chosen: together with the period of the lattice this thickness determines the position and width of the bandgap. Conversely, the incident angle determines the periodicity and hence also the properties of the gap. Calculations were used to optimize the layer thicknesses and the angle of incidence in order to obtain a photonic bandgap between 600 and $700 \mathrm{~nm}$ while eliminating unwanted reflections. To have sufficient flexibility in the optimization process, we introduced a $\mathrm{SiO}_{2}$ layer between the $\mathrm{Si}_{3} \mathrm{~N}_{4}$ and the $\mathrm{Si}$. A last goal in the optimization process was to gain a maximum degree of insensitivity to small variations in the layer thickness. The optimal layer stack was found to consist of a $3.2 \mu \mathrm{m}$ thick $\mathrm{SiO}_{2}$ layer between a thin $(150 \mathrm{~nm}) \mathrm{Si}_{3} \mathrm{~N}_{4}$ layer and a $\mathrm{Si}$ substrate. It has an optimal reflection behavior for a wide thickness range $(100 \mathrm{~nm})$ of the $\mathrm{Si}_{3} \mathrm{~N}_{4}$ layer around $150 \mathrm{~nm}$.

The layer stack was fabricated as follows. The thick $\mathrm{SiO}_{2}$ layer is formed by thermal oxidation of a $\mathrm{Si}$ wafer. On top of the $\mathrm{SiO}_{2}$ layer the $\mathrm{Si}_{3} \mathrm{~N}_{4}$ layer is grown by low-pressure chemical vapor deposition (LPCVD). The thickness of both layers is checked by ellipsometry. When necessary the thickness is adjusted with a slow wet etch. Then, a $300 \mathrm{~nm}$ photo resist layer is spin coated onto the $\mathrm{Si}_{3} \mathrm{~N}_{4}$.

In the next step of the procedure (Fig. 1, step C) the triangular dot pattern is generated in the photo resist with LIL. The dot pattern is subsequently used for a chromium lift off. Thus, a highly selective etch mask with a hole pattern is fabricated. The lift off is performed by evaporating a $20 \mathrm{~nm}$ thin layer of chromium after which the photo resist dots are dissolved in acetone using an ultrasonic bath agitation.

In order to prevent collapse of the freestanding $\mathrm{Si}_{3} \mathrm{~N}_{4}$ slabs, the large area (about $1.5 \mathrm{~cm}^{2}$ ) has to be divided into smaller sections. The length of the freestanding sections is defined by etching stripes of the chromium in a wet etch process. Conventional mask lithography is used to deposit an etch mask of $100 \mu \mathrm{m}$ wide bars of $1.2 \mu \mathrm{m}$ thick photo resist to define the supports of the photonic crystal sections (step D in Fig. 1). The photo resist will prevent the conversion of the chromium hole pattern into the $\mathrm{Si}_{3} \mathrm{~N}_{4}$ layer underneath the photo resist bars. The absence of holes will result in $\mathrm{SiO}_{2}$ supports when the freestanding slabs are created by etching. The supports define the width of the photonic crystal slab. The LIL-exposed area can yield a few hundred photonic crystal sections of $100 \mu \mathrm{m} \times 1$ to $4 \mathrm{~mm}$.

In the crystal sections defect structures were realized by covering some holes in the chromium etch mask (step E in Fig. 1). A local and additional platinum etch mask is created by FIB assisted deposition. ${ }^{[11]}$ The nanometer resolution of the FIB allows an accurate alignment of the deposition with respect to the LIL lattice. Because the periodic structure is already produced with the LIL, so-called stitching errors of the FIB are completely absent. An FIB of accelerated $\mathrm{Ga}^{+}$ ions, with $7 \mathrm{~nm}$ spot size, is scanned over the chromium hole structure in the presence of a platinum bearing gas ((trimethyl)-methylcyclopenta-dienylplatinum, $\left(\mathrm{CH}_{3}\right)_{3}\left(\mathrm{CH}_{3}\right.$ $\left.\mathrm{C}_{5} \mathrm{H}_{4}\right) \mathrm{Pt}$ ), which decomposes in the presence of the ion beam, resulting in a local deposition of $\mathrm{Pt}^{\left[{ }^{[2]}\right.}$ Platinum of $50 \mathrm{~nm}$ thickness suffices to act as an etch mask. The FIB deposition combines high accuracy and flexibility on the nanometer scale, which allows us to introduce any kind of defect structure. Figure $2 \mathrm{~b}$ depicts a chromium mask with a FIB-deposited platinum mask for a line defect. At this stage the FIB could in principle be used to modify the actual size of some of the holes in the Cr mask, either by local deposition of Pt or by local sputtering of the Cr mask. Presently, it is not clear what the quality of the resulting holes after etching (next fabrication step) might be.
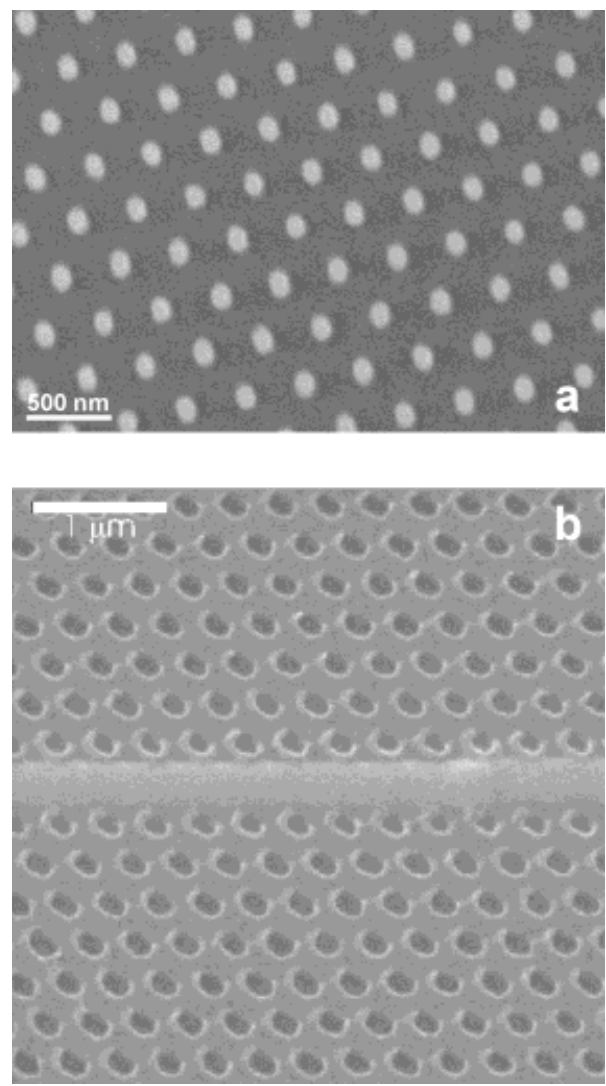

Fig. 2. a) SEM image of triangular photo resist dot pattern with a period of $300 \mathrm{~nm}$ after LIL. The uniform pattern extends over an area of the order of a centimeter squared. b) FIB image of an approximately $50 \mathrm{~nm}$ thick platinum line covering holes in a $20 \mathrm{~nm}$ thin chromium mask. The platinum line defines a defect structure in the photonic crystal slab and is deposited locally with a FIB.

The next step in the procedure (step F in Fig. 1) is a reactive ion etch (RIE) to transfer the composite mask pattern into the $\mathrm{Si}_{3} \mathrm{~N}_{4}$ layer. Then all mask materials are removed: the platinum and chromium masks in two wet etch steps, the photo resist in an oxygen plasma. A highly selective wet etch through the holes in the $\mathrm{Si}_{3} \mathrm{~N}_{4}$ etches the $\mathrm{SiO}_{2}$ layer below the crystal slab sections, so they become freestanding. In the absence of holes, where the $\mathrm{Si}_{3} \mathrm{~N}_{4}$ was protected by photo resist in the RIE process, the $\mathrm{SiO}_{2}$ layer remains intact and forms support bars between the sections. With a length and width of $6 \mathrm{~mm}$ and $200 \mu \mathrm{m}$, respectively, the $\pm 150 \mathrm{~nm}$ thick slabs did 
not collapse. The distance between the slab and the $\mathrm{Si}$ substrate is determined by the thickness of the original $\mathrm{SiO}_{2}$ layer $(3.2 \mu \mathrm{m})$. This distance is easily sufficient to confine the light in the slab and guarantee the desired mirror symmetry.

Figure $3 \mathrm{a}$ is a scanning electron microscopy (SEM) image of the end face of a $146 \mathrm{~nm}$ thick photonic crystal slab, which bridges $100 \mu \mathrm{m}$ between the support bars over a length of $4 \mathrm{~mm}$. The high uniformity of the periodic pattern extends along the entire area of the slab. In the middle of
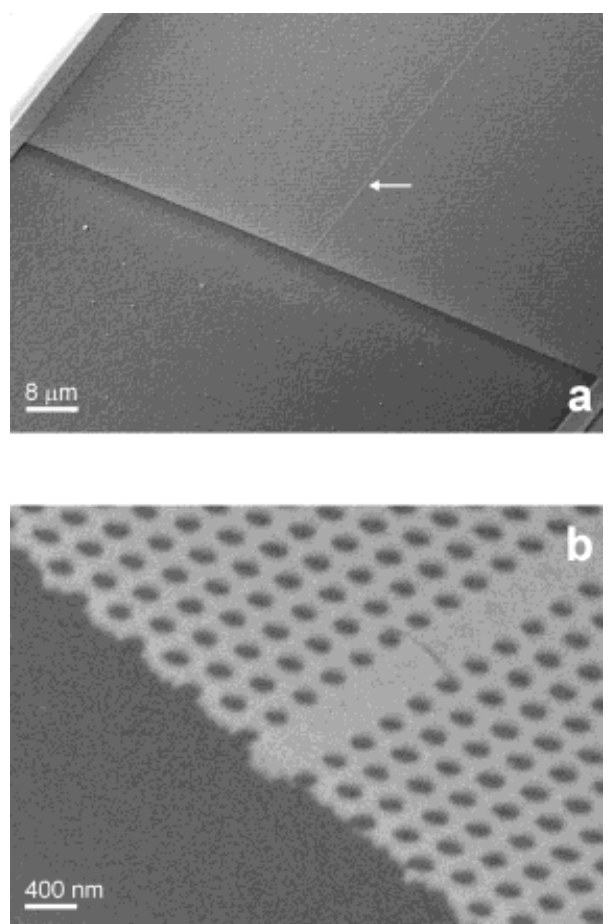

Fig. 3. a) SEM image of the end face of a photonic crystal slab between the support bars. This $146 \mathrm{~nm}$ thin slab is freestanding across $100 \mu \mathrm{m}$ and has a length of $4 \mathrm{~mm}$. The arrow indicates the location of a $1.1 \mathrm{~mm}$ line defect that runs parallel to the support bars along the center of the slab. b) SEM image, close-up of the defect line in the photonic crystal slab. The highly uniform triangular hole pattern has a period of $297 \mathrm{~nm}$. The axial widths of the elliptical holes are 212 and $164 \mathrm{~nm}$. The shape of the holes appears unharmed by the presence of the defect line. The defect is expected to form a multimode waveguide for visible light (estimated central wavelength $670 \mathrm{~nm}$ ).

the slab a line defect is produced parallel to the support bars. Figure $3 b$ depicts the start of the defect in greater detail. The length of the line defect is $1.1 \mathrm{~mm}$. The period of the triangular hole pattern is $297 \mathrm{~nm}$. The holes are slightly elliptical with axial widths of 212 and $164 \mathrm{~nm}$. The elliptical shape of the holes originates from the $60^{\circ}$ rotation in the double LIL exposure. ${ }^{[13]}$ The shape of the holes close to the defect line has not been influenced by the local platinum deposition. Preliminary calculations for this photonic crystal indicate the central wavelength of the bandgap to be at approximately $670 \mathrm{~nm}$ in air.

In conclusion, we have presented a novel method for the fabrication of extended photonic crystal slabs with lightguiding defects. We have manufactured highly uniform pho- tonic crystal slabs for visible light extending over areas of several hundred micrometer squared using LIL. The advantage of LIL is that the entire periodic pattern is created at once. We have used the high accuracy and flexibility of FIB-deposition to introduce line defects in the extended crystals. In principle, any defect is possible. The combination of LIL with FIB deposition opens avenues for all the major optical applications of photonic crystals. Due to its simplicity and scope, FIB-assisted LIL is a promising alternative for e-beam lithography in the fabrication of photonic materials.

\section{Experimental}

The LIL exposure was done on Olin 907/12 thinned with EEP (ethyl-3ethoxypropionate) and MMP (methyl-3-methoxypropionate), spin-coated at $4000 \mathrm{rpm}$ to form layers of $300 \mathrm{~nm}$ thickness. The exposure was performed with the $363.8 \mathrm{~nm}$ line of an argon laser.

The $\mathrm{SiO}_{2}$ was grown by thermal oxidation at $1150{ }^{\circ} \mathrm{C}$ for $29 \mathrm{~h}$ and annealed for $1 \mathrm{~h}$. The Si-rich $\mathrm{Si}_{3} \mathrm{~N}_{4}$ was grown by liquid-phase chemical vapor deposition (LPCVD) of $\mathrm{NH}_{3}$ and $\mathrm{SiH}_{2} \mathrm{Cl}_{2}$ at 250 mtorr and $850^{\circ} \mathrm{C}$ for $29 \mathrm{~min}$. The thickness of the layers was monitored with a Woollam ellipsometer. The measured refractive index is 2.15 at a wavelength between 600 and $700 \mathrm{~nm}$. When necessary the thickness of the $\mathrm{Si}_{3} \mathrm{~N}_{4}$ was adjusted by a HF (50\%) wet etch. Platinum was locally deposited with a FEI FIB 200 series at a beam current of $4 \mathrm{pA} / \mathrm{um}^{2}$.

RIE of the $\mathrm{Si}_{3} \mathrm{~N}_{4}$ was performed in a $\mathrm{CHF}_{3} / \mathrm{O}_{2}$ plasma at a forward power of $50 \mathrm{~W}$ in an Electrotech PF 320 system. The wet etch to remove the platinum consisted of water, $\mathrm{HCl}$, and fuming $\mathrm{HNO}_{3}(3: 7: 1)$ at a temperature of $85^{\circ} \mathrm{C}$. For the removal of the chromium we used a standard wet etch consisting of $\mathrm{HCl}$ $60 \%, \mathrm{Ce}\left(\mathrm{NH}_{4}\right)_{2}\left(\mathrm{NO}_{3}\right)_{6}$, and $\mathrm{H}_{2} \mathrm{O}$. Etching of the underlying $\mathrm{SiO}_{2}$ layer was performed by a wet etch in buffered HF. All SEM images were made with an ISI SEM.

Received: March 29, 2001 Final version: June 26, 2001

[1] See, for example, J. D. Joannopoulos, R. D. Meade, J. N. Winn, Photonic Crystals, Molding the Flow of Light, Princeton University Press, Princeton, NJ 1995.

[2] E. Yablonovitch, Phys. Rev. B 1987, 58, 2059.

[3] M. Loncar, T. Doll, J. Vuckovic, A. Scherer, J. Lightwave Technol. 2000, $18,1402$.

[4] T. F. Krauss, R. M. De La Rue, S. Brand, Nature 1996, 383, 699.

[5] S. G. Johnson, S. Fan, P. R. Villeneuve, J. D. Joannopoulos, Phys. Rev. B 2000, 60, 5751 .

[6] See for example, M. D. B. Charlton, M. E. Zoorob, G. J. Parker, M. C. Netti, J. J. Baumberg, S. Cox, H. Kemhadjian, Mater. Sci. Eng. B: Solid State Mater. Adv. Technol. 2000, 74, 17.

[7] V. Berger, O. Gauthier-Lafaye, E. Costard, Electron. Lett. 1997, 33, 425.

[8] C. J. M. van Rijn, G. J. Veldhuis, S. Kuiper, Nanotechnology 1998, 9, 343.

[9] M. Campbell, D. N. Sharp, M. T. Harrison, R. G. Denning, A. J. Turberfield, Nature 2000, 404, 53.

[10] C. Peeters, E. Flück, A. M. Otter, M. L. M. Balistreri, J. P. Korterik, L. Kuipers, N. F. van Hulst, Appl. Phys. Lett. 2000, 77, 142

[11] J. Orloff, Rev. Sci. Instrum. 1993, 64, 1105.

[12] A. J. DeMarco, J. Melngailis, J. Vac. Sci. Technol. B 1999, 17, 3154.

[13] S. C. Kitson, W. L. Barnes, J. R. Sambles, IEEE Photon. Technol. Lett. 1996, 8,1662 